\title{
Métodos de avaliação da qualidade de assistência ao pré-natal no Brasil: revisão integrativa da literatura
}

\author{
Methods of evaluating the quality of prenatal care in Brasil: integrative literature \\ review
}

\author{
Métodos de evaluación de la calidad de asistencia al prenatal en Brasil: revisión \\ integrativa de la literature
}

Gisselle Cascaes da Cruz ${ }^{1}$, Patrícia Carvalho Ruiz ${ }^{1}$, Orácio Carvalho Ribeiro Junior ${ }^{1 *}$, Adriana Duarte de Sousa ${ }^{1}$, Rhuana Maria de Oliveira Pereira ${ }^{1}$, Carolina Oldenburg Barroso ${ }^{1}$, Soraia Santos Tatikawa Campos ${ }^{1}$.

\section{RESUMO}

Objetivo: Analisar o perfil dos métodos de avaliação da qualidade de assistência ao pré-natal no Brasil Métodos: Trata-se de uma revisão integrativa da literatura com busca por artigos científicos disponíveis nas bases de dados Scientific Electronic Library Online (SCIELO) e Literatura Latino-Americana em Ciências da Saúde (LILACS) e Literatura Internacional em Ciências da Saúde (MEDLINE) no período de janeiro de 2012 a setembro de 2018. Resultados: Através de 10 artigos analisados foi possível identificar que as avaliações da atenção ao pré-natal se deram por diferentes métodos, alguns com escopo próprio e validado, outros tendo por base as diretrizes do Ministério da Saúde para a organização do pré-natal. Considerações finais: Percebe-se que há diversos parâmetros para a avaliação do pré-natal no Brasil, todos com enfoque quantitativo, sendo necessária a construção de novos métodos que sejam qualitativos que consigam avaliar aspectos da assistência ainda marginalizados, trazendo um panorama mais real da atenção pré-natal no país.

Palavras-chave: Cuidado pré-natal, Qualidade da assistência à saúde, Avaliação em saúde, Atenção à saúde.

\section{ABSTRACT}

Objective: To analyze the profile of prenatal care quality assessment methods in Brazil Methodology: This is an integrative review of the literature with search for scientific articles available in the Scientific Electronic Library Online (SCIELO) and Latin Literature databases American Journal of Health Sciences (LILACS) and International Literature on Health Sciences MEDLINE from January 2012 to September 2018. Results: Through 10 articles analyzed it was possible to identify that prenatal care different methods, some with their own validated scope, others based on the guidelines of the Ministry of Health for the organization of prenatal care. Final considerations: It is noticed that there are several parameters for prenatal evaluation in Brazil, all of them with a quantitative approach, and it is necessary to construct new qualitative methods that can evaluate aspects of assistance that are still marginalized, bringing a more real prenatal care in the country.

Keywords: Prenatal care, Quality of health care, Health assessment, Health care.

${ }^{1}$ Centro Universitário do Norte (UNINORTE), Manaus- AM. *E-mail: o_ra_cio13@hotmail.com

SUBMETIDO EM: 2/2019

ACEITO EM: $3 / 2019$

PUBLICADO EM: 7/2019

REAS/EJCH | Vol. Sup.27 | e521 | DOI: https://doi.org/10.25248/reas.e521.2019 Página 1 de 8 


\section{RESUMEN}

Objetivo: El objetivo de este trabajo es analizar el perfil de los métodos de evaluación de la calidad de asistencia al prenatal en Brasil. Métodos: Se trata de una revisión integrativa de la literatura con búsqueda de artículos científicos disponibles en las bases de datos Scientific Electronic Library Online (SCIELO) y Literatura Latino (LILACS) y Literatura Internacional en Ciencias de la Salud (MEDLINE) en el período de enero de 2012 a septiembre de 2018. Resultados: Através de 10 artículos analizados fue posible identificar que las evaluaciones de la atención al prenatal si se de acuerdo con las directrices del Ministerio de Salud para la organización del prenatal. Consideraciones finales: Se percibe que hay diversos parámetros para la evaluación del prenatal en Brasil, todos con enfoque cuantitativo, siendo necesaria la construcción de nuevos métodos que sean cualitativos que consigan evaluar aspectos de la asistencia aún marginados, trayendo un panorama más real de la atención prenatal en el país.

Palabras clave: Cuidado prenatal, Calidad de la asistencia sanitaria, Evaluación en salud, Atención a la salud.

\section{INTRODUÇÃO}

Nos últimos anos a assistência à saúde da mulher no Brasil tem ampliado as ações e serviços de promoção da saúde e prevenção de agravos, favorecendo essa população específica e reduzindo iniquidades sociais, como a mortalidade materna e neonatal no país.

Em 1984, o Ministério da Saúde criou o Programa de Assistência Integral à Saúde da Mulher (PAISM), que inclui a assistência à mulher em clínica ginecológica, pré-natal, parto e puerpério, e no climatério.

Já em 2011 este escopo de ações e serviços é ampliado e o programa ganha status de política, passando a denominar-se de Política Nacional de Atenção Integral à Saúde da Mulher (PNAISM) que passou a dar fundamento a todas as ações voltadas à saúde da mulher no país (BRASIL, 2011).

Como estratégia primária para a redução de morbimortalidade em mulheres no ciclo gravídico-puerperal por causas preveníveis, foram criados o Programa de Humanização do Pré-Natal e Nascimento (PHPN) e mais tarde o Programa Rede Cegonha, que dentre outros enfoques, estabeleceram a qualificação da expansão e principalmente da qualidade da atenção ao pré-natal no Brasil.

Estes programas e politicas públicas de saúde voltados à saúde da mulher permitiram uma expansão da cobertura de atenção ao pré-natal, que hoje atinge o percentual de cobertura de mais de $90 \%$ em todas as regiões do país, contribuindo para a redução da razão de mortalidadade materna nos últimos anos (BRASIL, 2002; BRASIL, 2011).

Sabendo-se que a atenção ao pré-natal de qualidade é capaz de diminuir a morbidade e a morbimortalidade materno infantil, o atendimento de gestantes para o início do pré-natal é essencial para o acompanhamento, pois auxilia no diagnóstico precoce de alterações e para a realização de condutas adequadas sobre condições que a gestante pode ser acometida (BRASIL, 2016; TOMASI E, et al., 2017).

Embora seja bastante extensa a literatura que mostra a importância da assistência ao pré-natal de forma qualificada no contexto da saúde da mulher, tem-se observado que, apesar da larga expansão de cobertura dos serviços de pré-natal no país nos últimos anos, o mesmo não aconteceu com a qualidade de atenção destas ações. Estudos revelam que a qualidade da assistência ao pré-natal no Brasil tem apresentado várias fragilidades de conteúdo e que leva por consequência ao aparecimento de complicações na vida desta mulher, tais como: doenças hipertensivas, síndromes hemorrágicas e infecção do trato urinário (NUNES JT, et al., 2016).

Neste contexto observam-se vários estudos que tem por finalidade realizar avaliação da qualidade de atenção aos serviços de assistência ao pré-natal com diferentes parâmetros, ligados sobretudo às recomendações do Ministério da Saúde elou a algum parâmetro específico e já validado por autores consagrados na literatura sobre a temática (SILVA EP, et al., 2013a). 
Por tanto, torna-se relevante traçar um panorama de métodos de avaliação propostos por diferentes estudos no Brasil, para que se possa ter de forma fidedigna a completude e a correlação desses métodos de avaliação com a propositura da assistência que é proposta pelo Ministério da Saúde, podendo então diferir se tais métodos são adequáveis para a realidade do país.

Desta forma, este estudo tem por objetivo analisar o perfil dos métodos de avaliação da qualidade de assistência ao pré-natal no Brasil.

\section{MÉTODOS}

Trata-se de um estudo do tipo revisão integrativa da literatura, conduzida a partir de artigos publicados no período de janeiro de 2012 a setembro de 2018, com foco a análise de pesquisas realizadas pelos autores independentes, que foram abordados métodos de avaliação, onde verifica-se a qualidade da atenção ao prénatal no país produzida a partir de 6 fases, conforme preconiza a literatura de base sobre este tipo de estudo (TEIXEIRA et al., 2013).

A seleção dos artigos foi realizada através de busca nas bases de dados LILACS (Literatura LatinoAmericana e do Caribe em Ciências da Saúde), MEDLINE (Literatura Internacional em Ciência da Saúde) e SCIELO (Scientific Electronic Library Online).

Para realizar as buscas foram utilizados os seguintes descritores: "Cuidado pré-natal", "Qualidade da assistência à saúde", "Avaliação em saúde", "Atenção à saúde". Todos disponíveis nos Descritores em Ciências da Saúde (DeCS).

A partir da filtragem dos artigos forma estabelecidos os critérios de inclusão, sendo selecionados os artigos que estavam em texto completo, publicados no Brasil, em idioma português, que compreendiam o período proposto e que atendessem os objetivos da revisão.

Os critérios de exclusão foram: monografia, teses, dissertações, relatos de experiências e artigos repetidos nas bases de dados.

A análise dos dados foi feita através da elaboração de um quadro que organizou cada artigo selecionado contendo: Base, Revista, Título, Autores, Objetivo, Parâmetros de avaliação de qualidade, Metodologia e ano.

\section{RESULTADOS}

Inicialmente foram analisados 164 artigos nas bases: Literatura Latino-Americana em Ciências da Saúde (LILACS), Scientific Electronic Library Online (SCIELO) e MEDLINE - Literatura Internacional em Ciências da Saúde, anos publicados posterior à 2012, e após aplicarmos os critérios de inclusão e exclusão forma selecionados 10 artigos.

Todos os artigos que foram selecionados têm como interesse buscar de que forma estão acontecendo as avaliações de assistência do pré-natal no Brasil, utilizando critérios e métodos para verificar se a qualidade da assistência está prevalecendo.

Com base nos artigos obteve-se um conhecimento amplo, onde observa-se a magnitude de avaliações e buscas de uma qualidade da assistência do pré-natal, sendo assim, foi criado um quadro contendo: base, revista, título, autor(es), objetivo, parâmetro de avaliação da qualidade, metodologia e ano.

A revisão mostra ainda que todos os estudos estão no escopo transversal ou quanti-qualitativo, conforme evidenciando no quadro 1. 


\section{Revista Eletrônica Acervo Saúde / Electronic Journal Collection Health ～ISSN 2178-2091}

\begin{tabular}{|c|c|c|c|c|c|c|c|c|c|}
\hline \multirow[t]{2}{*}{ № } & \multirow[t]{2}{*}{ Base } & \multirow[t]{2}{*}{ Revista } & \multirow[t]{2}{*}{ Título } & \multirow[t]{2}{*}{ Autor (es) } & \multirow[t]{2}{*}{ Objetivo } & \multirow{2}{*}{$\begin{array}{c}\text { Parâmetro de } \\
\text { avaliação da } \\
\text { qualidade. }\end{array}$} & \multicolumn{2}{|c|}{ Metodologia } & \multirow[t]{2}{*}{ Ano } \\
\hline & & & & & & & Tipo de estudo & Abordagem & \\
\hline 1 & LILAC & $\begin{array}{l}\text { Caderno } \\
\text { de saúde } \\
\text { pública, } \\
\text { Rio de } \\
\text { Janeiro }\end{array}$ & $\begin{array}{l}\text { Avaliação da adequação da } \\
\text { assistência pré-natal na } \\
\text { rede SUS do Município do } \\
\text { Rio de Janeiro, Brasil }\end{array}$ & $\begin{array}{l}\text { Domingues RMSM } \\
\text { et al. }\end{array}$ & $\begin{array}{l}\text { Objetivo deste estudo é avaliar } \\
\text { a adequação da assistência } \\
\text { pré-natal na rede do SUS do } \\
\text { Município do Rio de Janeiro }\end{array}$ & $\begin{array}{l}\text { Utilizado o índice } \\
\text { PHPN, e um índice } \\
\text { PHPN Ampliado, em } \\
\text { que foram } \\
\text { acrescentados } \\
\text { procedimentos clínicos } \\
\text { obstétricos, prescrição } \\
\text { de sulfato ferroso } \\
\text { suplementar e ações } \\
\text { educativas. }\end{array}$ & Transversal & Quantitativo & 2012 \\
\hline 2 & SCIELO & $\begin{array}{l}\text { Rev. } \\
\text { Bras } \\
\text { Ginecol } \\
\text { Obst }\end{array}$ & $\begin{array}{l}\text { Qualidade da Assistência } \\
\text { pré-natal nos serviços } \\
\text { públicos e privados. }\end{array}$ & $\begin{array}{l}\text { Paris GF, Pelloso } \\
\text { SM, Martins PM. }\end{array}$ & $\begin{array}{l}\text { Analisar assistência pré-natal } \\
\text { nos serviços de saúde públicos } \\
\text { e privados. }\end{array}$ & $\begin{array}{l}\text { Foi baseado nas } \\
\text { recomendações do } \\
\text { PHPN, no percentual } \\
\text { de não registro de uma } \\
\text { variável }\end{array}$ & Transversal & Quantitativa & 2013 \\
\hline 3 & SCIELO & $\begin{array}{l}\text { Rev. } \\
\text { Escola } \\
\text { de } \\
\text { Enfermag } \\
\text { em USP. }\end{array}$ & $\begin{array}{l}\text { Avaliação da Assistência } \\
\text { pré-natal em Unidade com } \\
\text { Estratégia Saúde da } \\
\text { Família. }\end{array}$ & $\begin{array}{ll}\text { Corrêa } & M D, \\
\text { Tsunechiro } & M A, \\
\text { et.al. } & \end{array}$ & $\begin{array}{l}\text { Analisar o processo da } \\
\text { assistência Pré-natal de uma } \\
\text { unidade com Estratégia Saúde } \\
\text { da Família. }\end{array}$ & $\begin{array}{l}\text { Conforme três } \\
\text { indicadores do PHPN, } \\
\text { início até } 16 \text { semanas, } \\
\text { mínimo de seis } \\
\text { consultas, realização } \\
\text { de consulta puerperal. }\end{array}$ & Transversal. & Quantitativa & 2014 \\
\hline 4 & LILAC & $\begin{array}{l}\text { Rev. } \\
\text { Brasileira } \\
\text { de Saúde } \\
\text { Materno } \\
\text { Infantil. }\end{array}$ & $\begin{array}{l}\text { Avaliação da qualidade da } \\
\text { atenção pré-natal dentre } \\
\text { gestantes com e sem } \\
\text { história de pré maturidade } \\
\text { do sistema único de saúde } \\
\text { no RJ, Brasil. }\end{array}$ & $\begin{array}{l}\text { Vettore MV, Dias M, } \\
\text { et al. }\end{array}$ & $\begin{array}{l}\text { Avaliar adequação, } \\
\text { acompanhamento pré-natal, } \\
\text { satisfação e risco gestacionais } \\
\text { das gestantes com história de } \\
\text { pré maturidade. }\end{array}$ & $\begin{array}{l}\text { Qualidade da } \\
\text { assistência do pré- } \\
\text { natal foi avaliada com } \\
\text { índice de Kotelchuck. }\end{array}$ & Seccional. & Quantitativa & 2013 \\
\hline 5 & SCIELO & $\begin{array}{l}\text { Rev. } \\
\text { Brasileira } \\
\text { de Saúde } \\
\text { Materno } \\
\text { Infantil. }\end{array}$ & $\begin{array}{l}\text { Pré-natal em mulheres } \\
\text { usuárias do sistema único } \\
\text { de saúde em duas } \\
\text { maternidades no estado do } \\
\text { RJ, Brasil: a cor importa? }\end{array}$ & $\begin{array}{l}\text { Fonseca SC, Kale } \\
\text { PL, Silva KS. }\end{array}$ & $\begin{array}{l}\text { Identificar fatores associados } \\
\text { ao pré-natal inadequado, com } \\
\text { destaque para a cor da pele, } \\
\text { em usuárias do SUS do estado } \\
\text { do RJ. }\end{array}$ & $\begin{array}{l}\text { Baseado nas } \\
\text { adaptações nacionais } \\
\text { do índice de } \\
\text { Kotelchuck. }\end{array}$ & Transversal & Quantitativa & 2015 \\
\hline
\end{tabular}

REAS/EJCH | Vol. Sup.27 | e521 | DOI: https://doi.org/10.25248/reas.e521.2019 Página 4 de 8 


\section{Revista Eletrônica Acervo Saúde / Electronic Journal Collection Health ～ISSN 2178-2091}

\begin{tabular}{|c|c|c|c|c|c|c|c|c|c|}
\hline 6 & SCIELO & $\begin{array}{l}\text { Rev. } \\
\text { Brasileira } \\
\text { de Saúde } \\
\text { Materno } \\
\text { Infantil. }\end{array}$ & $\begin{array}{l}\text { Pré-natal na atenção } \\
\text { primaria do município de } \\
\text { João } \\
\text { Caracterização de serviços } \\
\text { e usuárias. }\end{array}$ & $\begin{array}{l}\text { Silva EP, Lima RT, } \\
\text { Ferreira NLS, Costa } \\
\text { MJC }\end{array}$ & $\begin{array}{l}\text { Caracterizar a assistência pré- } \\
\text { natal em unidade básica de } \\
\text { saúder envolvendo } \\
\text { profissionais e usuárias do } \\
\text { município de João Pessoa-PB }\end{array}$ & $\begin{array}{l}\text { A classificação do pré- } \\
\text { natal em categoria de } \\
\text { adequação foi feita a } \\
\text { partir dos critérios } \\
\text { definidos pelos Índices } \\
\text { de Kessner e Índice de } \\
\text { Adequação da } \\
\text { Utilização do Cuidado } \\
\text { Pré-natal (APNCU) }\end{array}$ & $\begin{array}{l}\text { Transversal } \\
\text { Analítico- } \\
\text { descritivo }\end{array}$ & Quantitativa & 2013 \\
\hline 7 & LILAC & $\begin{array}{l}\text { Caderno } \\
\text { de saúde } \\
\text { pública, } \\
\text { Rio de } \\
\text { Janeiro. }\end{array}$ & $\begin{array}{lrr}\text { Assistência } & \text { pré-natal } & \text { nos } \\
\text { serviços } & \text { públicos } & \text { e } \\
\text { privados de saúde: } & \text { estudo } \\
\text { transversal de } & \text { base } \\
\text { populacional } & \text { em } & \text { Rio } \\
\text { Grande do Sul, Brasil. } & \end{array}$ & Cesar AJ et al & $\begin{array}{l}\text { Estimar a cobertura para } \\
\text { diversos exames laboratoriais, } \\
\text { procedimentos clínicos e } \\
\text { orientações recebidas durante } \\
\text { o pré-natal }\end{array}$ & $\begin{array}{l}\text { Baseado nos Critérios } \\
\text { de Takeda e Silveira e } \\
\text { colaboradores. }\end{array}$ & Transversal & Quantitativa & 2012 \\
\hline 8 & LILAC & $\begin{array}{l}\text { Caderno } \\
\text { de saúde } \\
\text { pública, } \\
\text { Rio de } \\
\text { Janeiro }\end{array}$ & $\begin{array}{l}\text { O que os cartões de pré- } \\
\text { natal das gestantes } \\
\text { revelam sobre a assistência } \\
\text { nos serviços do SUS da } \\
\text { Região Metropolitana da } \\
\text { Grande Vitória, Espírito } \\
\text { Santo, Brasil }\end{array}$ & $\begin{array}{l}\text { Santos Neto ET, } \\
\text { Oliveira AE, et al }\end{array}$ & $\begin{array}{l}\text { Propõe avaliar a completude } \\
\text { de informações sobre } \\
\text { Assistência pré-natal nos } \\
\text { cartões de gestantes. }\end{array}$ & $\begin{array}{l}\text { Parâmetros de Romero } \\
\text { \& Cunha, foi criado um } \\
\text { escore a partir da } \\
\text { incompletude. }\end{array}$ & Transversal & $\begin{array}{l}\text { Qualitativa, } \\
\text { quantitativa }\end{array}$ & 2012 \\
\hline 9 & MEDLINE & $\begin{array}{l}\text { Caderno } \\
\text { de saúde } \\
\text { pública, } \\
\text { Rio de } \\
\text { Janeiro }\end{array}$ & $\begin{array}{l}\text { Qualidade da atenção pré- } \\
\text { natal na rede básica de } \\
\text { saúde do Brasil: } \\
\text { indicadores } \\
\text { desigualdades sociais }\end{array}$ & $\begin{array}{l}\text { Tomasi } \quad \text { E., } \\
\text { Fernandes P.A.A, } \\
\text { Fischer T., et al }\end{array}$ & $\begin{array}{l}\text { Descrever indicadores de } \\
\text { qualidade da atenção pré- } \\
\text { natal no Brasil no âmbito do } \\
\text { Programa de Melhoria do } \\
\text { Acesso e da Qualidade } \\
\text { (PMAQ-AB). }\end{array}$ & $\begin{array}{l}\text { Parâmetro próprio, } \\
\text { construído com base } \\
\text { nas diretrizes do } \\
\text { Ministério da Saúde } \\
\text { para o pré-natal de } \\
\text { baixo risco através dos } \\
\text { dados da avaliação } \\
\text { externa do I ciclo do } \\
\text { PMAQ-AB. }\end{array}$ & Transversal & $\begin{array}{l}\text { Qualitativo } \\
\text { quantitativo }\end{array}$ & 2017 \\
\hline 10 & LILAC & $\begin{array}{l}\text { Revista } \\
\text { Pan- } \\
\text { American } \\
\text { a de } \\
\text { Pública }\end{array}$ & $\begin{array}{l}\text { Desenvolvimento } \\
\text { aplicação de um novo } \\
\text { Índice para avaliação do } \\
\text { pré-natal Saúde }\end{array}$ & $\begin{array}{l}\text { Silva EP, Lima RT, } \\
\text { Costa MJC, Batista } \\
\text { Filho M. }\end{array}$ & $\begin{array}{l}\text { Desenvolvimento e a aplicação } \\
\text { de um novo instrumento para } \\
\text { avaliar o pré-natal de forma } \\
\text { mais abrangente. }\end{array}$ & $\begin{array}{l}\text { Índice Infraestrutura, } \\
\text { Processo e } \\
\text { Resultados, IPR/pré- } \\
\text { natal, baseado nas } \\
\text { diretrizes do PHPN }\end{array}$ & Transversal & $\begin{array}{l}\text { Quantitativo } \\
\text { Qualitativo }\end{array}$ & 2013 \\
\hline
\end{tabular}

Fonte: Autores, 2019.

REAS/EJCH | Vol. Sup.27 | e521 | DOI: https://doi.org/10.25248/reas.e521.2019 Página 5 de 8 


\section{DISCUSSÃO}

Os estudos referentes à avaliação da qualidade da atenção ao pré-natal no Brasil mostram que os métodos empregados para determinar o fator qualidade das ações e serviços do referido programa são essencialmente baseados no programa de humanização do pré-natal e nascimento (PHPN) que determina no país a conformação de um pré-natal adequado. Outros parâmetros também têm sido empregados, como o Índice de Kessner e os indicadores do programa nacional de melhoria do acesso e qualidade da atenção básica (PMAQ), porém, todos estes remetem aos critérios já fundamentos pelo Ministério da Saúde no âmbito do PHPN.

Segundo estudo realizado por Domingues RMSM et al. (2012) no município do Rio de Janeiro, os autores avaliaram a adequação da assistência ao pré-natal segundo as recomendações do PHPN: início do pré-natal, número mínimo de consultas para a idade gestacional, resultado da $1^{\underline{a}}$ rotina de exames, vacina antitetânica, e resultado da $2^{\text {a }}$ rotina de exames; considera-se também um índice PHPN ampliado, onde neste se acrescentam durante as consultas, aferição de pressão arterial e peso, registro da idade gestacional, medida da altura uterina, ausculta de batimentos cardiofetais, assim como prescrição de sulfato ferroso suplementar e orientações sobre o parto e amamentação. Ambos conforme a semana gestacional recomendado pelo PHPN. A assistência ao pré-natal foi considerada adequada quando a gestante apresentou os procedimentos previstos de acordo com a idade gestacional.

Já no estudo realizado por Paris GF et al. (2013), na região dos Campos Gerais- Estado do Paraná, os autores avaliaram a qualidade de assistência ao pré-natal nos serviços públicos e privados, com base no percentual dos não registros de uma variável, seguindo as recomendações do PHPN, onde estratificaram a conformidade em: excelente, bom, regular, ruim e muito ruim. Excelente: quando o percentual de não registro foi de 5\%; bom: quando o percentual de não registro foi de 5 a $9 \%$; regular: quando o percentual de não registro foi de 10 a 19\%; ruim: de 20 a 49\%; e muito ruim: quando o percentual de não registro foi igual ou superior a 50\%. E no estudo proposto por Correa MD et al. (2014), desenvolvido em unidade com Estratégia Saúde da Família do município de São Paulo, a qualidade da assistência ao pré-natal foi avaliada conforme três indicadores do PHPN: com início até 16 semanas, mínimo de seis consultas, e realização de consulta puerperal, que estratificaram em adequado as que obedeceram a esses critérios e inadequado para as demais.

Em outros estudos desenvolvido por Vettore MV et al. (2013) e Fonseca SC et al. (2015), ambos no Rio de Janeiro, a qualidade da assistência Pré-natal foi avaliada conforme o índice de Kotelchuck que estratifica em mais que adequado, adequado, intermediário e inadequado, considerando-se em: inadequado a realização de menos de $50 \%$ das consultas esperadas no início do pré-natal após o quarto mês de gestação, em intermediário com $50-79 \%$, adequado $80-109 \%$ e mais do que adequado com a realização de $\geq 110 \%$ das consultas esperadas, com característica demográfica e socioeconômica: idade, raça/cor da pele, escolaridade, situação conjugal, e atividade renumerada. Em uma particularidade no estudo desenvolvido por Fonseca SC et al. (2015) com destaque para a cor da pele, as demais categorias intermediárias, adequado, e mais que adequado, foram agrupadas em uma única categoria, classificadas como adequada.

Outro estudo de análise da adequação da assistência ao pré-natal no município de João Pessoa, utilizouse o índice de Kessner que relaciona a semana de início do pré-natal, a idade gestacional no parto e o número de visitas de pré-natal classificando em adequado, intermediário e inadequado.

Considera-se: pré-natal iniciado antes da $13^{a}$ semana gestacional com uma ou mais consultas, duas ou mais consultas para a gestação de 14 a 17 semanas, três ou mais para gestação de 18 a 21 semanas e assim por diante, tendo como inadequado caso não haja consulta ou número de consulta muito inferior ao esperado para a idade gestacional, e intermediário para as demais combinações; e também utilizou o índice de Adequação da Utilização do Cuidado Pré-natal (APNCU) proposto por Kotelchuck (SILVA EP, et al., 2013b).

O Índice de Kessner foi um dos primeiros índices criados para avaliar a qualidade da assistência ao prénatal em 1973, modificado posteriormente em 1994 por Kotelchuck, e no Brasil em 1993 Takeda propôs um novo índice baseado no índice de Kessner. 
Em 2001, Silveira e colaboradores modificaram este índice acrescentando ao pré-natal adequado, a realização de hemograma, exame de urina e sorológico para sífilis (SAAVEDRA JS e CESAR JA, 2015).

Já Cesar JA et al. (2012), em seu estudo realizado em Rio Grande do Sul, propôs avaliar a assistência ao pré-natal nos serviços públicos e privados, de acordo com os critérios de Takeda e o índice de Kessner modificado por Silveira, onde ambos estabelecem três categorias: Adequado, Inadequado e Intermediário. Takeda relaciona o número total de consultas com o ingresso ao pré-natal, considerando: adequado para seis ou mais consultas antes de cinco meses de gestação, inadequado menos de três consultas e se estas ocorrem após o sétimo mês gestacional, e intermediário para as demais situações.

Na Região Metropolitana da Grande Vitória-ES, a qualidade da assistência ao pré-natal foi avaliada através dos cartões das gestantes. Neste estudo Santos Neto ET et al. (2012), usam os critérios de completude de Romero \& Cunha para a devida avaliação. Estratificaram o nível da qualidade em: excelente (incompletude $<5 \%$ ), bom (incompletude $>5 \%$ e $<10 \%$ ), regular (incompletude $\geq 10 \%$ e $<20 \%$ ), ruim (incompletude $\geq 20 \%$ e $<50 \%$ ) e muito ruim (incompletude $\geq 50 \%$ ), de acordo com as variáveis do PHPN.

Já em estudo realizado no Brasil com os dados gerados pelo Programa de Melhoria do acesso e da Qualidade da Atenção Básica, PMAQ-AB, a qualidade da assistência ao pré-natal foi avaliada de acordo com os indicadores: número de consultas, situação vacinal, prescrição de sulfato ferroso, exame físico, orientações, e exames complementares, de acordo com o protocolo Ministerial para pré-natal de baixo risco. Foi considerada com qualidade adequada quando a mulher afirmou ter realizado no mínimo seis consultas durante a gestação, todos os cincos exames complementares, vacina antitetânica (se necessário), recebido prescrição de sulfato ferroso, ter recebido os cincos procedimentos do exame físico e as orientações (TOMASI E, et al., 2017).

Em um estudo desenvolvido no município de João Pessoa no ano de 2013, os autores desenvolveram e aplicaram um novo índice para avaliação de pré-natal com base nas diretrizes do Ministério da Saúde, o instrumento elaborado continha 23 questões relativas à Infraestrutura, Processo de trabalho, e Resultados da assistência ao pré-natal com elementos quanti-qualitativos, o qual denominaram de índice IPR/Pré-natal. Neste índice os autores atribuem 1 para adequado e 2 para inadequado, considerando as questões de análises para os itens "Infraestrutura" seis questões, "Processo de trabalho" seis questões com metas propostas pelo PHPN, e no item "Resultados" 11 questões conforme os critérios do PHPN. A avaliação foi feita a partir da soma do número das 23 questões adequadas dos três itens, que estratificaram a exemplo de Kessner e Kotelchuck, em adequada superior, adequada, intermediário e inadequado. Quanto maior a soma, mais elevada se torna o grau de adequação (SILVA EP, et al., 2013a).

Percebe-se que a atenção ao pré-natal no Brasil tem sido avaliada principalmente por parâmetros do Ministério da Saúde, variando em alguns estudos com algumas modificações, que acompanham as ações e serviços que têm sido acrescentados ao escopo do programa em questão ao longo do tempo. Acredita-se que estes parâmetros são adequados para avaliar a qualidade da atenção ao pré-natal, pois, procuram determinar quais ações, daquelas preconizadas pelo estado brasileiro, de fato estão acontecendo nos diferentes contextos assistenciais. Contudo, não se deve excluir a necessidade de verificação da qualidade do referido programa junto aos profissionais de saúde e às usuárias que são os principais atores do processo.

\section{CONSIDERAÇÕES FINAIS}

A avaliação da qualidade da atenção pré-natal no Brasil tem se dado sob parâmetros quantitativos validados e diversificados sendo os baseados no PHPN os mais empregados. Outros métodos como o índice de Kessner, Kotelchuck, Takeda e Silveira têm sido empregados nos estudos de avaliação da qualidade, todos estes ligados as diretrizes do Ministério da Saúde. Porém, percebe-se que há uma necessidade da criação de novos métodos qualitativos que permitam a avaliação da qualidade da assistência ao pré-natal, uma vez que os métodos supracitados propõem essencialmente uma avaliação quantitativa da assistência, podendo ocorrer a ocultação de outros aspectos fundamentais que qualificam o pré-natal e seus derivantes, sendo necessário novos critérios que possam mensurar de forma integral a qualidade da assistência. 


\section{REFERÊNCIAS}

1. BRASIL. Ministério da Saúde (BR), Secretaria Executiva. Humanização do parto: humanização no pré-natal e nascimento. Brasília: Ministério da Saúde; 28 p, 2002.

2. BRASIL. Ministério da Saúde. Protocolos da Atenção Básica: Saúde das Mulheres/ Ministério da Saúde, Instituto SírioLibanês de Ensino e Pesquisa - Brasília: Ministério da Saúde, 230 p, 2016.

3. BRASIL. Política Nacional de Atenção Integral à Saúde da Mulher: Princípios e Diretrizes/Ministério da Saúde, Secretaria de Atenção à Saúde, Departamento de Ações Programáticas Estratégicas. - 1. ed., 2. Brasília: Editora do Ministério da Saúde, 44 p, 2011.

4. CESAR JA, et al. Assistência pré-natal nos serviços públicos e privados de saúde: estudo transversal de base populacional em Rio Grande, Rio Grande do Sul, Brasil. Cadernos de Saúde Pública, 2012; 28(11): 2106-2114.

5. CORREA MD, et al. Avaliação da assistência pré-natal em unidade com estratégia saúde da família. Revista da Escola de Enfermagem da USP, 2014; 48(Esp): 24-32.

6. DOMINGUES RMSM, et al. Avaliação da adequação da assistência pré-natal na rede SUS do Município do Rio de Janeiro, Brasil. Cadernos de Saúde Pública, 2012; 28(3): 425-437.

7. FONSECA SC, et al. Pré-natal em mulheres usuárias do Sistema Único de Saúde em duas maternidades no Estado do Rio de Janeiro, Brasil: a cor importa?. Revista Brasileira de Saúde Materno-Infantil, 2015; 15 (2): $209-217$.

8. NUNES JT, et al. Qualidade da assistência pré-natal no Brasil: revisão de artigos publicados de 2005 a 2015 . Cadernos de Saúde Coletiva, 2016; 24 (2): 252-261.

9. PARIS GF, et al. Qualidade da assistência pré-natal nos serviços públicos e privados. Revista Brasileira de Ginecologia e Obstetricia, 2013; 35(10): 447-52.

10. SAAVEDRA JS, CESAR JA. Uso de diferentes critérios para avaliação da inadequação do pré-natal: um estudo de base populacional no extremo Sul do Brasil. Cadernos de Saúde Pública, 2015; 31(5):1003-1014.

11. SANTOS NETO ET, et al. O que os cartões de pré-natal das gestantes revelam sobre a assistência nos serviços do SUS da Região Metropolitana da Grande Vitória, Espírito Santo, Brasil?. Cadernos de Saúde Pública, 28(9): 1650-1662. 12. SILVA EP, et al. Desenvolvimento e aplicação de um novo índice para avaliação do pré-natal. Revista Panamericana de Saúde Pública, 2013a; 33(5): 356-362.

13. SILVA EP, et al. Pré-natal na atenção primária do município de João Pessoa-PB: caracterização de serviços e usuárias. Revista Brasileira de Saúde Materno-Infantil, 2013; 13 (1): 29-37.

14. TEIXEIRA E, et al. Revisão Integrativa da Literatura passo-a-passo \& convergências com outros métodos de revisão. Revista Enfermagem UFPI, 2013; 2(spe): 3-7.

15. TOMASI E, et al. Qualidade da atenção pré-natal na rede básica de saúde do Brasil: indicadores e desigualdades sociais. Cadernos de Saúde Pública, 2017; 33(3): 1-11.

16. VETTORE MV, et al. Avaliação da qualidade da atenção pré-natal dentre gestantes com e sem história de prematuridade no Sistema Único de Saúde no Rio de Janeiro, Brasil. Revista Brasileira de Saúde Materno-Infantil, 2013; 13 (2): 89-100. 